\title{
Autoécologie des chênaies de Quercus robur L. en Galice (Espagne)
}

\author{
Ignacio Javier DíAZ-MAROTOa*, Pablo VILA-LAMEIROa ${ }^{a}$, Francisco Javier SILVA-PANDOb,c \\ a Departamento de Enxeñería Agroforestal, Escola Politécnica Superior, Campus Universitario s/n, Universidade de Santiago de Compostela, \\ 27002 Lugo, Espagne \\ b Centro de Investigacións Forestais e Ambientais de Lourizán, Consellería de Medio Ambiente, Apdo 127, 36080 Pontevedra, Espagne \\ c Departamento de Producción Vexetal, Escola Politécnica Superior, Campus Universitario s/n, Universidade de Santiago de Compostela, \\ 27002 Lugo, Espagne
}

(Reçu le 30 mars 2004 ; accepté le 14 février 2005)

\begin{abstract}
Résumé - Une caractérisation paramétrique des biotopes occupés actuellement par des formations de " carballo » (Quercus robur L.) est présentée pour la Galice (Espagne). Pour ceci, on a inventorié un total de 39 parcelles et on a élaboré 28 paramètres écologiques caractéristiques du biotope, avec 14 autres paramètres qui caractérisent de façon dendrométrique et sylvicole les chênaies inventoriées. Cette information a permis de définir l'habitat physiographique, climatique et édaphique, central et marginal, de Quercus robur en Galice, ainsi que d'établir la caractérisation de la situation actuelle de ses massifs d'un point de vue dendrométrique et sylvicole. Les chênaies galiciennes présentent une hétérogénéité élevée aussi bien dans les biotopes où elles se situent que dans l'utilisation et la gestion auxquelles elles ont été soumises. La variation altitudinale est supérieure à $1200 \mathrm{~m}$, ceci se traduit par une différence de température moyenne de $7,3^{\circ} \mathrm{C}$. Les précipitations moyennes annuelles sont de $1371,9 \mathrm{~mm}$, la moyenne estivale atteint $164 \mathrm{~mm}$, en conséquence, l'aridité estivale est très faible. Les sols, en majorité des cambisols, sont fortement acides, avec une composition texturale à dominante sableuse, ceci est dû à la nature des matériaux géologiques (granites et schistes) desquels ils proviennent.
\end{abstract}

Quercus robur / autoécologie / biotope / limite écologique / Galice

\begin{abstract}
Autoecology of oaks (Quercus robur L.) in Galicia (Spain). A parametric characterization of the biotypes currently in stands of "carballo" (Quercus robur L) in Galicia (Spain) is presented. For this, an inventory of a total of 39 plots has been made. Twenty-eight ecological parameters characteristic of each biotype as well as 14 more to characterize forest mensuration and silviculture of studied oaklands were elaborated. This information has permitted the determination of the fisiographic, climatic and edaphic habitat of Quercus robur in central and marginal area in Galicia, as well as the characterisation of the actual situation from a silvicultural stands point of view. Galician oakwoods are highly heterogeneous, as well in biotype location as their use and management they had been subjected. Their altitudinal difference is near to $1200 \mathrm{~m}$ and this signify a mean temperature difference of $7.3{ }^{\circ} \mathrm{C}$. The annual precipitation average is $1371.9 \mathrm{~mm}$; with $164 \mathrm{~mm}$ in summer rains, in consequence summer dryness is very scarce. The soils, mainly cambisols, are strongly acidic, with a dominant sandy textural composition due to the nature of their geological origin, which are by and large granite and schist rocks.
\end{abstract}

Quercus robur / autoecology / site / ecologic limit / Galicia

\section{INTRODUCTION}

En Galice, la végétation climacique de la majeure partie du territoire serait occupée aujourd'hui par différentes espèces de Quercus faisant partie des forêts caducifoliées galiciennes [37, 53]. Selon les analyses palynologiques, ces forêts devinrent dominantes entre cinq et sept milles ans après la dernière glaciation du Quaternaire, lorsque le climat est devenu plus humide et chaud [52]. Les forêts de Quercus robur L., seraient les formations climaciques qui occuperaient une grande partie du territoire galicien, depuis le niveau de la mer jusqu' aux altitudes de 1300-1400 m, constituées par différentes espèces ou formant des communautés diverses qui s'accommodent aux variétés climatiques présentes. Le climat atlantique dominant sur la majeure partie de Galice est idéal pour le développement d'une chênaie mixte avec la présence de bouleau (Betula celtiberica) et de châtaignier (Castanea sativa) [28], qui sont normalement accompagnés d'autres espèces comme le noisetier (Corylus avellana), le houx (Ilex aquifolium), etc., et de différentes espèces arbustives et herbacées, constituant, d'un point de vue phytosociologique, diverses associations inclues dans l'ordre Quercetalia robori-petraea [1, 37, 53, 60].

Ou peut distinguer une chênaie acidophile (Blechno spicanti-Quercetum roboris Tx. \& Oberdofer 1958) caractéristique de la zone nord de Galice. Dans la moitié occidentale, audessous des 550-600 m, on trouve un autre type de chênaie humide ou hyper humide et acidophile (Rusco aculeati-Quercetum roboris Br.-Bl., P. Silva \& Rozeira 1956), et dans des localités

* Auteur pour correspondance : diazmaro@lugo.usc.es 
situées entre 500-1500 m avec une période de sécheresse estivale non prononcée ; à l'intérieur de la Galice, ainsi qu'au sud, dans des localités possédant une plus grande continentalité, et parfois, avec l'influence du climat méditerranéen, apparaît aussi une chênaie humide ou hyper humide (Vaccinio myrtilliQuercetum roboris P. Silva, Rozeira \& Fontes 1950) [37, 60].

La majeure partie de ces bois naturels sont très dégradés, il s'agit de forêts qui n'ont reçu aucun traitement sylvicole, ou celui qu'ils ont reçu fut, dans la majorité des cas, malencontreux (jardinage pied par pied des meilleurs exemplaires et têtards des arbres) [35, 42]. Les profits abusifs séculiers, le pâturage excessif, le défrichage des forêts, les grands incendies du passé et les actuels... ont amené les chênaies atlantiques galiciennes à la situation actuelle $[6,18,28,60]$. Les mieux conservées se trouvent dans des vallées escarpées ou des zones écartées, dans lesquelles d'autres mises en valeur du sol sont difficiles. On note l'apparition fréquente de chênes et de châtaigniers centenaires de grand diamètre, mais très fréquemment mal formés et pourris $[27,28]$.

La grande demande sociale pour la conservation et la récupération de ces formations est un des aspects où l'on constate un changement d'attitude comme le reflète l'élaboration du Plan Forestier de Galice en 1992, qui proposait d'augmenter considérablement la superficie occupée par ces forêts. Par ailleurs, dans la procédure établie pour la création du Réseau Natura 2000 de l'Union Européenne, Directive 92/43/CEE, on souligne, plus particulièrement, le besoin d'inclure comme habitat d'intérêt communautaire les chênaies galiciennes et portugaises de Quercus robur et Quercus pyrenaica. Donc le besoin de conserver les chênaies actuellement existantes et d'augmenter leur zone d'occupation actuelle semble évident, tout en évitant les risques par une gestion sylvicole inadéquate. C'est la raison pour laquelle, la connaissance de l'autoécologie de ces formations est indispensable [7, 18, 38, 40].

Les exigences écologiques des chênaies galiciennes de Quercus robur ne sont pas suffisamment connues. Izco et al. [37] et Silva-Pando [60] ont réalisé une révision phytosociologique détaillée de ces forêts pour toute la Galice, alors que Izco et al. [38] présentent une étude phytosociologique dans le nord de la province de A Coruña (Galice). D'autres auteurs comme $[8,11,45,46]$, entre autres, ont recueilli des données phytosociologiques ou écologiques plus ou moins étendues de ces forêts dans différentes zones de Galice. Diaz-Maroto [18] a réalisé une étude écologique et dendrométrique des massifs naturels de chêne et [6] a étudié leur croissance et leur production à l'intérieur du territoire galicien. Comme territoires proches de la Galice, nous possédons les études réalisées dans le nord du Portugal [14] et dans les Asturies et Cantabria (Espagne) $[15,23]$. Concernant d'autres territoires, nous remarquerons, parmi d'autres, les travaux [7, 40, 44, 54].

Pour avancer dans la connaissance des exigences écologiques de Quercus robur en Galice, il est nécessaire de descendre au niveau régional, pour cela, nous prétendons caractériser le fonctionnement des écosystèmes de Quercus robur à partir de divers paramètres écologiques mettant en relation ceux-ci avec les paramètres dendrométriques et sylvicoles, indicateurs de l'usage et de l'état de conservation de ces formations $[5,9,23$, $24,55]$.

\section{MATÉRIEL ET MÉTHODES D'ÉTUDE}

\subsection{Domaine d'étude}

La zone étudiée comprend toute la Communauté Autonome de Galice (Espagne) (Fig. 1). Selon les données du $3^{\mathrm{e}}$ Inventaire Forestier National, la superficie occupée en Galice par les forêts de feuillus autochtones est de 375922 ha (approximativement $27 \%$ de la superficie totale boisée) ; si à cette donnée nous ajoutons, d'une part, la superficie occupée par des mélanges de conifères et de feuillus, 337008 ha et, d'autre part, la superficie correspondante aux mélanges de feuillus autochtones et d'Eucalyptus globulus, 12285 ha, on atteint une superficie totale de 725215 ha, c'est-à-dire, presque $52 \%$ de la superficie totale boisée de Galice [20]. L'importance de ces données est plus grande si ou prend en compte leur évolution, car en 1986, les forêts de feuillus autochtones occupaient uniquement $20 \%$, un pourcentage qui s'élevait jusqu'à $38 \%$ si on lui ajoutait les mélanges de conifères et feuillus [65]. Dans ce contexte, les forêts pures de Quercus robur occupent une superficie de 187789 ha, presque $14 \%$ de la superficie totale boisée de Galice, même si cette espèce apparaît également mélangée à d'autres feuillus autochtones (Betula celtiberica, Corylus avellana, Acer pseudoplatanus, Quercus pyrenaica, Quercus petraea, ...), à Pinus pinaster et à Eucalyptus globulus, avec une superficie sensiblement supérieure $[6,20]$.

\subsection{Caractères écologiques de Quercus robur en Galice}

Il s'agit d'une espèce typique de la région eurosibérienne qui abonde dans la majeure partie de la Galice, à l'exception des zones d'influence méditerranéenne, possédant un climat de précipitations sub-humide à hyper humide, mais qui nécessite une certaine quantité de pluie et d'humidité, aussi bien atmosphérique qu'édaphique pendant l'été. En Galice, elle reçoit de 800 jusqu'à plus de $3000 \mathrm{~mm} / \mathrm{an}$ de précipitations totales. Elle résiste au froid, en étant présente jusqu'à $1300 \mathrm{~m}$ d'altitude $[1,12,17,18]$. Pour son développement normal, elle nécessite des sols profonds, frais et meubles, même si elle supporte quelques inondations temporaires. Les sols sur lesquels s'implante le Quercus robur dans la zone étudiée sont, en général, de type cambisol, même s'il s'implante aussi sur des fluvisols ; dans tous les cas le $\mathrm{pH}$ est acide [16]. Les substrats prédominants sont divers (granite, schiste, gneiss, quartz et ardoise) $[12,18,60]$. Du point de vue physiographique, elle se situe particulièrement dans des zones plates ou de coteaux à pente douce, surtout dans de larges vallées fraîches. Dans les coteaux, elle préfère les expositions tempérées aux plus chaudes ou froides. C'est une espèce au tempérament robuste, exigeante en lumière, elle ne tolère pas l'ombre dans les premières phases de son développement. Dans le domaine d'étude, on peut la considérer comme une espèce de mi-lumière $[12,17,60]$.

\subsection{Description de l'échantillonnage et de la prise de données}

Dans un premier temps, on envisagea de réaliser une stratification du domaine d'étude [9, 24,25], cependant, dans notre cas, ceci n'était pas réalisable, étant donné la dispersion et l'hétérogénéité des chênaies sur le territoire galicien. C'est pour cela, que nous avons considéré toute la zone à étudier comme une unité mais en éliminant certaines localités où les particularités du biotope rendent énormément difficile la présence de Quercus robur $[18,60]$. Pour cela, en prenant comme point de départ l'information recueillie sur la carte forestière d'Espagne [56], nous avons délimité le domaine d'étude et avons sélectionné les zones pour échantillonnages d'une manière subjective en nous basant sur les données apportées par le personnel de l'administration, et sur l'information provenant de travaux antérieurs [13, 18]. En tout nous avons choisi 39 parcelles (Tab. I). 


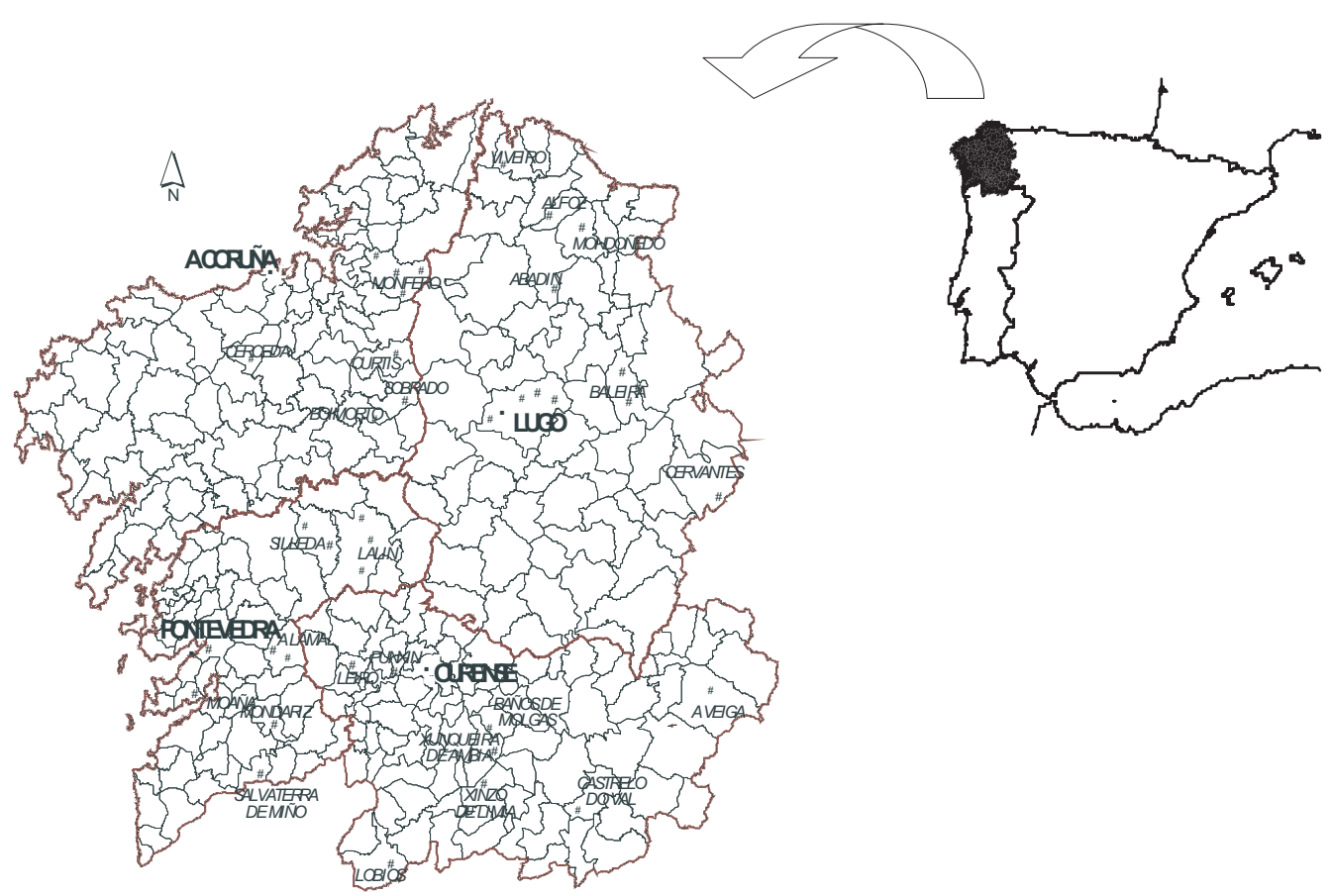

Figure 1. Situation du domaine d'étude (Communauté Autonome de Galice) dans le contexte de l'Espagne. Localisation par provinces des points d'échantillonnage.

Pour sélectionner le lieu idéal de situation de chaque parcelle dans la zone d'échantillonnage, nous avons réalisé au préalable la reconnaissance et l'analyse des conditions existantes du milieu pour pouvoir choisir le lieu de caractéristiques moyennes qui définissent cette zone $[11,23]$. Suivant une tendance aujourd'hui généralisée des divers auteurs et parmi eux Guitián Rivera [26], nous avons opté pour l'implantation de parcelles carrées de $10 \mathrm{~m}$ de côté. Nous avons étudié 3 parcelles de $100 \mathrm{~m}^{2}$ dans chacune des zones inventoriées, avec l'objectif d'échantillonner de manière plus précise, la possible hétérogénéité de chaque localité. Nous avons étudié les caractéristiques physiographiques, dendrométriques et le profil du sol, identifiant et décrivant les différents horizons édaphiques. Avec les données de l'inventaire des parcelles, les données climatologiques adaptées aux différents points d'échantillonnage selon la méthodologie de Carballeira et al. [10], et les résultats des analyses physiques et chimiques des échantillons de sol, nous avons élaboré un ensemble de paramètres caractéristiques des habitats physiographiques, climatiques et édaphiques de Quercus robur en Galice, ainsi que de l'état dendrométrique et sylvicole des chênaies [5, 9, 24, 25, 55].

\subsection{Paramètres élaborés (voir annexe)}

Nous avons élaboré un total de 42 paramètres, 28 paramètres écologiques descriptifs du biotope $[11,23,24,40,55,62]$ et des conditions d'environnement qui déterminent la régénération et conditionnent la croissance des chênaies [4, 36, 41, 44] et 14 qui caractérisent de manière dendrométrique et sylvicole les forêts étudiées $[18,62]$.

Les paramètres physiographiques qui décrivent chacune des zones étudiées ont été $[11,18,55]$ : l'altitude moyenne, la pente moyenne, la profondeur du sol jusqu'à la roche mère et la distance à la mer.

Le climat de chacune des zones a été décrit selon les paramètres suivants $[44,51]$ : précipitations totales annuelles, précipitations estivales, température moyenne annuelle, température moyenne annuelle des maximas absolus et température moyenne annuelle des minimas absolus.
Nous avons considéré 19 paramètres édaphiques, les trois premiers évaluent les caractéristiques physiques du sol, les huit suivants évaluent les propriétés chimiques et les huit restants leur fertilité $[9,11$, $23-25,55]$ : sable total, limon total, argile totale, $\mathrm{pH}$ total $\mathrm{H}_{2} \mathrm{O}, \mathrm{pH}$ superficiel $\mathrm{H}_{2} \mathrm{O}$, matière organique totale, matière organique superficielle, azote total, azote superficiel, rapport $\mathrm{C} / \mathrm{N}$ total, rapport $\mathrm{C} / \mathrm{N}$ superficiel, phosphore assimilable total, phosphore assimilable superficiel, potassium échangeable total, potassium échangeable superficiel, calcium échangeable total, calcium échangeable superficiel, magnésium échangeable total et magnésium échangeable superficiel. Lorsque nous considérons la valeur totale du paramètre dans l'ensemble du profil du sol, nous prenons une moyenne pondérée standard de tout le profil édaphique pour le sable, le limon et l'argile ; pour le reste, nous prenons la moyenne pondérée de tout le profil édaphique appliquant la méthode de Russell et Moore [57]. Pour les valeurs superficielles des paramètres édaphiques nous prenons la donnée des $20 \mathrm{~cm}$ supérieurs du sol $[23,24,55]$, sauf lorsque, dans cette épaisseur, il existe plus d'un horizon. Il est alors nécessaire de calculer une moyenne pondérée.

À l'intérieur de chaque point d'échantillonnage nous avons calculé les paramètres dendrométriques/sylvicoles suivants $[6,18,48]$ : nombre de pieds par hectare, surface terrière par hectare, diamètre moyen arithmétique, diamètre moyen quadratique, déviation standard de la distribution diamétrique, coefficient de variation de la distribution diamétrique, diamètre dominant, hauteur moyenne arithmétique, hauteur moyenne quadratique, déviation standard de la distribution de hauteurs, coefficient de variation de la distribution de hauteurs, hauteur dominante de Assmann [2, 59], indice de Hart [29, 59] et indice de Czarnowski (ICZ), comme nombre de pieds existants dans une parcelle carrée dont la longueur est la hauteur moyenne arithmétique.

\subsection{Analyse statistique}

La base de données sur le biotope et la structure dendrométrique et sylvicole des chênaies de Quercus robur en Galice fut, en un premier 
Tableau I. Identification des parcelles d'échantillonnage avec indication du chiffre, nom, code, province où se elles se trouvent et données physiographiques (altitude, pente, orientation).

\begin{tabular}{|c|c|c|c|c|c|c|}
\hline $\begin{array}{l}\text { Numéro de } \\
\text { parcelle }\end{array}$ & $\begin{array}{l}\text { Nom de } \\
\text { parcelle }\end{array}$ & $\begin{array}{l}\text { Code de } \\
\text { parcelle }\end{array}$ & Province & $\begin{array}{l}\text { Altitude } \\
(\mathrm{m})\end{array}$ & $\begin{array}{c}\text { Pente } \\
(\%)\end{array}$ & Orientation \\
\hline 1 & Cerceda & $\mathrm{C}$ & A Coruña & 420 & 17 & Nord \\
\hline 2 & Cerqueiros I & CEI & A Coruña & 393 & 55 & Nord-ouest \\
\hline 3 & Lourizán & $\mathrm{L}$ & Pontevedra & 60 & 8 & Nord-est \\
\hline 4 & San Miguel de Bacurín & $\mathrm{B}$ & Lugo & 500 & 0 & - \\
\hline 5 & Ancares & A & Lugo & 1075 & 60 & Nord \\
\hline 6 & A Pena & AP & Ourense & 650 & 0 & - \\
\hline 7 & A Rúa & $\mathrm{AR}$ & Pontevedra & 240 & 14 & Nord \\
\hline 8 & Baños de Molgas & $\mathrm{BM}$ & Ourense & 565 & 10 & Nord-ouest \\
\hline 9 & Barcía & BA & Pontevedra & 740 & 12 & Sud-ouest \\
\hline 10 & Boimente & $\mathrm{BO}$ & Lugo & 510 & 8 & Nord-est \\
\hline 11 & Cerqueiros II & CEII & A Coruña & 400 & 60 & Est \\
\hline 12 & Coiró & $\mathrm{CO}$ & Pontevedra & 320 & 20 & Nord-est \\
\hline 13 & Curtis & $\mathrm{CU}$ & A Coruña & 480 & 27 & Sud \\
\hline 14 & Fragavella & $\mathrm{F}$ & Lugo & 620 & 48 & Sud-est \\
\hline 15 & Gomaríz & G & Ourense & 220 & 27 & Nord \\
\hline 16 & Lagoa & LA & Lugo & 110 & 8 & Sud-ouest \\
\hline 17 & Lobios & LO & Ourense & 790 & 53 & Nord \\
\hline 18 & Monfero & MO & A Coruña & 500 & 48 & Nord-ouest \\
\hline 19 & Monte Marronda I & MI & Lugo & 800 & 72 & Nord-est \\
\hline 20 & Monte Marronda II & MII & Lugo & 670 & 58 & Nord-est \\
\hline 21 & Monte Marronda III & MIII & Lugo & 540 & 60 & Nord-est \\
\hline 22 & Monte San Fitoiro I & MFI & Lugo & 620 & 0 & - \\
\hline 23 & Monte San Fitoiro II & MFII & Lugo & 630 & 15 & Nord-ouest \\
\hline 24 & Monte San Fitoiro III & MFIII & Lugo & 690 & 25 & Nord \\
\hline 25 & Ourantes & $\mathrm{O}$ & Ourense & 380 & 65 & Nord-est \\
\hline 26 & Ribeiro & RI & A Coruña & 360 & 21 & Sud-est \\
\hline 27 & Salvaterra do Miño & $\mathrm{S}$ & Pontevedra & 80 & 12 & Nord-est \\
\hline 28 & Serra do Candán I & SCI & Pontevedra & 540 & 38 & Nord-ouest \\
\hline 29 & Serra do Candán II & SCII & Pontevedra & 550 & 22 & Nord-ouest \\
\hline 30 & Serra do Candán III & SCIII & Pontevedra & 600 & 12 & Nord \\
\hline 31 & Serra do Invernadeiro & SD & Ourense & 1300 & 35 & Nord \\
\hline 32 & Siador & SI & Pontevedra & 600 & 27 & Nord-ouest \\
\hline 33 & Sobrado dos Monxes & SM & A Coruña & 520 & 0 & - \\
\hline 34 & Teixeiro & $\mathrm{T}$ & A Coruña & 300 & 10 & Est \\
\hline 35 & Valdín & VA & Ourense & 1080 & 36 & Est \\
\hline 36 & Viloalle & VI & Lugo & 265 & 23 & Sud-est \\
\hline 37 & Xesta & $\mathrm{X}$ & Pontevedra & 740 & 25 & Sud-ouest \\
\hline 38 & Xestoso & $\mathrm{XE}$ & Pontevedra & 600 & 19 & Nord-est \\
\hline 39 & Xunqueira de Ambía & $\mathrm{XU}$ & Ourense & 565 & 7 & Nord-ouest \\
\hline
\end{tabular}

temps, analysée statistiquement de manière univariable [63], en passant ensuite à la détermination des valeurs suivantes caractéristiques $[9,23-25,55]$ : limite inférieure (LI), valeur minimale du paramètre dans la totalité des points d'échantillonnage ; seuil inférieur (SI) : percentile 10 ; moyenne (M), valeur moyenne ; seuil supérieur (SS) : percentile 90 ; limite supérieure (LS), valeur maximale. En nous basant 


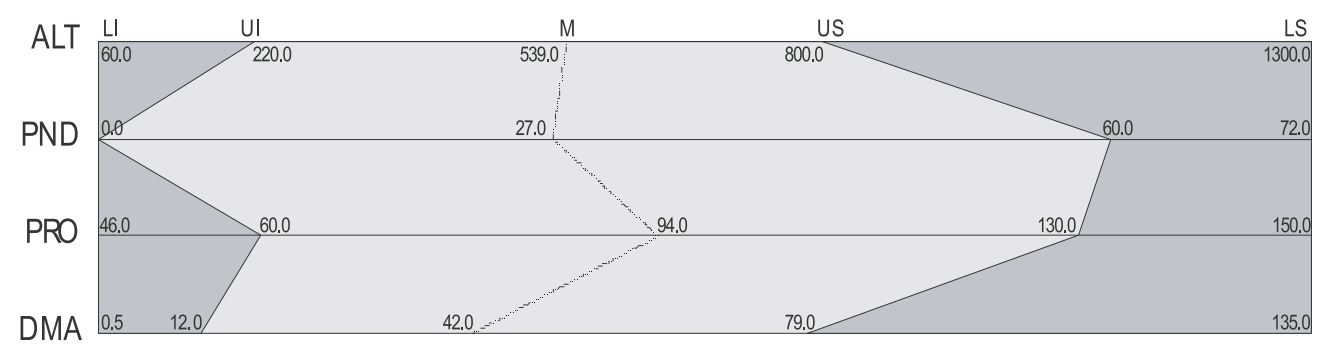

Figure 2. Habitat physiographique central et marginal des chênaies galiciennes de Quercus robur (voir liste des paramètres dans l'annexe).

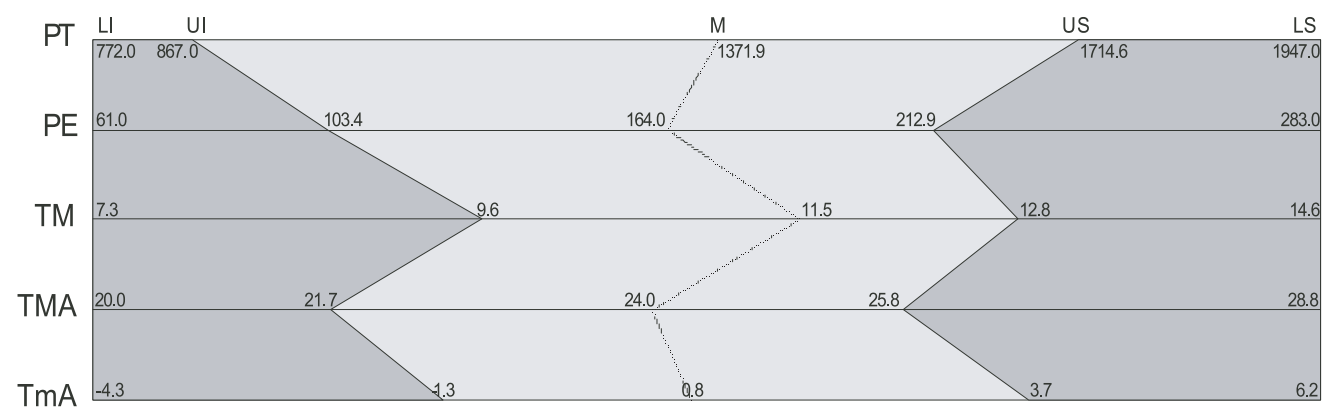

Figure 3. Habitat climatique central et marginal des chênaies galiciennes de Quercus robur (voir liste des paramètres dans l'annexe).

sur ces valeurs, il est possible de calculer les limites écologiques (physiographiques, climatiques et édaphiques) et de définir l'habitat des chênaies galiciennes de Quercus robur L., en accord avec les critères suivants [22-24] :

1. Qualifier comme habitat central ou optimum, par rapport à un paramètre, l'intervalle défini par les seuils supérieur et inférieur.

2. Considérer comme habitat marginal les intervalles compris entre la limite inférieure et le seuil inférieur et entre le seuil supérieur et la limite supérieure.

En principe, l'habitat central définit les conditions physiographiques, climatiques et édaphiques les plus aptes pour Quercus robur en Galice, alors que, dans l'habitat marginal, la condition non optimum de certains paramètres rend plus douteuse l'aptitude de la saison pour le chêne [23, 24]. Il faut dire, que les deux peuvent être seulement considérés comme une première approche pour décrire de façon adéquate la phytocoénose des forêts de chêne en Galice, car il est logique de penser que tous les paramètres élaborés n'auront pas la même importance ou signification comme descripteurs du milieu, mais que certains définiront avec une plus grande précision que d'autres la distribution de ces formations [22-24]. Pour cela, une fois déterminés ces habitats, il fut nécessaire de réaliser une analyse discriminante des parcelles, avec l'objectif d'identifier ces paramètres-là avec un plus grand poids descriptif [34, 43]. La méthode utilisée est celle proposée par Hill et al. $[30,33]$, qui permet d'arriver à une classification dichotomique de différentes parcelles, échantillonnages au hasard, en employant pour cela le programme Twinspan [31, 32, 49]. Le regroupement de parcelles se réalise en fonction de certains attributs de celles-ci, comme peuvent être la présence d'espèces botaniques déterminées $[6,24,33]$ des valeurs de paramètres écologiques et sylvicoles déterminés [3, 18, 24].

Chacun des paramètres classificateurs a été divisé en trois intervalles dont les limites sont établies de manière à ce que le nombre de parcelles inclu dans chacun de ceux-ci fût plus ou moins égal à un tiers du total, en nous basant sur les valeurs de leur moyenne et de la déviation standard [47]. Les limites de chaque intervalle ont été définies de la manière suivante $[18]$ :
Intervalle inférieur : $<$ Moyenne - Déviation standard $(<\mathrm{M}-\mathrm{DE})$; Intervalle moyen : Moyenne \pm Déviation standard $(>\mathrm{M}-\mathrm{DE} ;<$ $\mathrm{M}+\mathrm{DE})$;

Intervalle supérieur : $>$ Moyenne + Déviation standard $(>M+D E)$.

Ensuite, nous avons réalisé une analyse bivariable (matrice de corrélations) entre l'information sylvicole et les paramètres, aussi bien dendrométrique/sylvicoles, qu'avec les descripteurs du biotope [63]. L'ensemble de résultats de l'analyse de régressions bivariables rendra possible, à travers de l'extraction de composantes principales (ACP) $[21,39,61]$, l'identification de la manière dont les paramètres élaborés, expliquent la majeure partie de la variabilité possible de la population $[39,58,61]$.

\section{RÉSULTATS}

En se basant sur les données obtenues dans l'inventaire de parcelles et, postérieurement, des paramètres élaborés, nous disposons d'information pour caractériser l' habitat de Quercus robur en Galice. Dans le tableau II on recueille la description statistique univariable des paramètres du biotope et dans les figures 2, 3, et 4 sont représentés les habitats physiographique, climatique et édaphique.

\subsection{Habitats physiographiques et climatiques des chênaies galiciennes de $Q$. robur}

Suivant les critères notés par [23, 24], nous pouvons définir les habitats physiographiques et climatiques centraux, comme l'intervalle formé par $80 \%$ des parcelles étudiées et les habitats marginaux, compris entre les extrêmes absolus et les limites de l'intervalle central pour chacun des paramètres (Figs. 2 et 3). 
Tableau II. Moyennes, déviations standards (DE), coefficients de variation (CV), values maximales et minimales des paramètres physiographiques, climatiques et édaphiques des forêt de Quercus robur $(n=39)$. Les variables sans unités sont adimensionnelles.

\begin{tabular}{|c|c|c|c|c|c|}
\hline Paramètre & Moyenne & $\mathrm{DE}$ & $\mathrm{CV}(\%)$ & Maximale & Minimale \\
\hline $\operatorname{ALT}(\mathrm{m})$ & 539 & 260 & 48,2 & 1300 & 60 \\
\hline PND (\%) & 27 & 21 & 77,7 & 72 & 0 \\
\hline PRO (cm) & 94 & 26 & 27,6 & 150 & 46 \\
\hline $\mathrm{DM}(\mathrm{km})$ & 42 & 28 & 66,6 & 135 & 0,5 \\
\hline PT (mm) & $1.371,9$ & 311,6 & 22,7 & 1947,0 & 772,0 \\
\hline $\mathrm{PE}(\mathrm{mm})$ & 164,0 & 51,4 & 31,3 & 283,0 & 61,0 \\
\hline $\mathrm{TM}\left({ }^{\circ} \mathrm{C}\right)$ & 11,5 & 1,5 & 13,0 & 14,6 & 7,3 \\
\hline TMA $\left({ }^{\circ} \mathrm{C}\right)$ & 24,0 & 2,0 & 8,3 & 28,8 & 20,0 \\
\hline $\operatorname{TmA}\left({ }^{\circ} \mathrm{C}\right)$ & 0,8 & 2,4 & 300,0 & 6,2 & $-4,3$ \\
\hline $\mathrm{PH}$ & 4,85 & 0,46 & 9,3 & 6,15 & 3,92 \\
\hline $\mathrm{HS}$ & 4,71 & 0,51 & 10,7 & 6,53 & 3,82 \\
\hline $\mathrm{MO}(\%)$ & 8,64 & 5,19 & 60,0 & 23,31 & 1,04 \\
\hline $\operatorname{MOS}(\%)$ & 12,85 & 7,76 & 60,4 & 34,21 & 1,19 \\
\hline $\mathrm{N}(\%)$ & 0,307 & 0,178 & 58,0 & 0,793 & 0,042 \\
\hline NS (\%) & 0,442 & 0,232 & 52,5 & 1,019 & 0,050 \\
\hline $\mathrm{C} / \mathrm{N}$ & 14,6 & 4,5 & 31,0 & 29,6 & 6,9 \\
\hline $\mathrm{C} / \mathrm{NS}$ & 16,8 & 4,3 & 25,3 & 30,1 & 10,4 \\
\hline $\mathrm{P}(\mathrm{ppm})$ & 21,8 & 28,9 & 133,6 & 117,2 & 0,4 \\
\hline PS (ppm) & 19,8 & 29,2 & 147,5 & 119,5 & 0,4 \\
\hline $\mathrm{K}(\mathrm{ppm})$ & 73 & 40 & 54,8 & 231 & 9 \\
\hline KS (ppm) & 103 & 50 & 48,5 & 252 & 19 \\
\hline $\mathrm{Ca}(\mathrm{ppm})$ & 120 & 216 & 180,0 & 1297 & 3 \\
\hline $\mathrm{CaS}$ (ppm) & 170 & 285 & 167,6 & 1704 & 4 \\
\hline $\mathrm{Mg}(\mathrm{ppm})$ & 29 & 21 & 72,4 & 85 & 0 \\
\hline $\operatorname{MgS}(\mathrm{ppm})$ & 49 & 38 & 77,5 & 143 & 0 \\
\hline $\operatorname{ARE}(\%)$ & 65,78 & 20,50 & 31,2 & 88,67 & 13,83 \\
\hline LIM (\%) & 22,53 & 20,75 & 92,1 & 84,89 & 7,44 \\
\hline $\operatorname{ARC}(\%)$ & 11,22 & 5,09 & 45,4 & 26,82 & 1,27 \\
\hline
\end{tabular}

Voir liste des paramètres dans l'annexe.

\subsection{Habitat édaphique des chênaies galiciennes de $Q$. robur}

Dans la figure 4 nous avons défini l'habitat édaphique central et marginal des forêts de chêne en Galice. Dans cette figure, en analysant l'habitat édaphique marginal, nous pouvons observer l'énorme amplitude des intervalles marginaux supérieurs (seuil et limite) pour les paramètres $\mathrm{Ca}$ et $\mathrm{CaS}$. Ceci nous indique que ces caractéristiques du biotope ne sont pas de bonnes définitions, dans le cas des chênaies galiciennes, pour fixer la limite supérieure de l'habitat central [9, 24].

\subsection{Aspects dendrométriques et sylvicoles des chênaies galiciennes de $Q$. robur}

Les forêts étudiées présentent un grand éventail d'âges et de fertilité de stations $[6,18,27,28,60]$, ce qui a rendu difficile leur comparaison ; pour cela il n'a pas été possible de les caractériser d'un point de vue dendrométrique et sylvicole. Le tableau III, rapporte les statistiques descriptives de ces paramètres.

\subsection{Relation entre les paramètres dendrométriques/ sylvicoles et le biotope}

Une fois déterminé le biotope et les caractéristiques dendrométriques et sylvicoles, l'étape suivante a consisté à identifier ces paramètres écologiques avec un plus grand poids descriptif dans l'habitat de l'espèce [34] et, essayer de les mettre en relation avec les paramètres sylvicoles. Pour cela, nous avons réalisé une classification dichotomique des parcelles, en employant le programme Twinspan [31, 32, 49]. Nous n'avons pas limité le chiffre maximum de paramètres à utiliser dans l'analyse, donc 


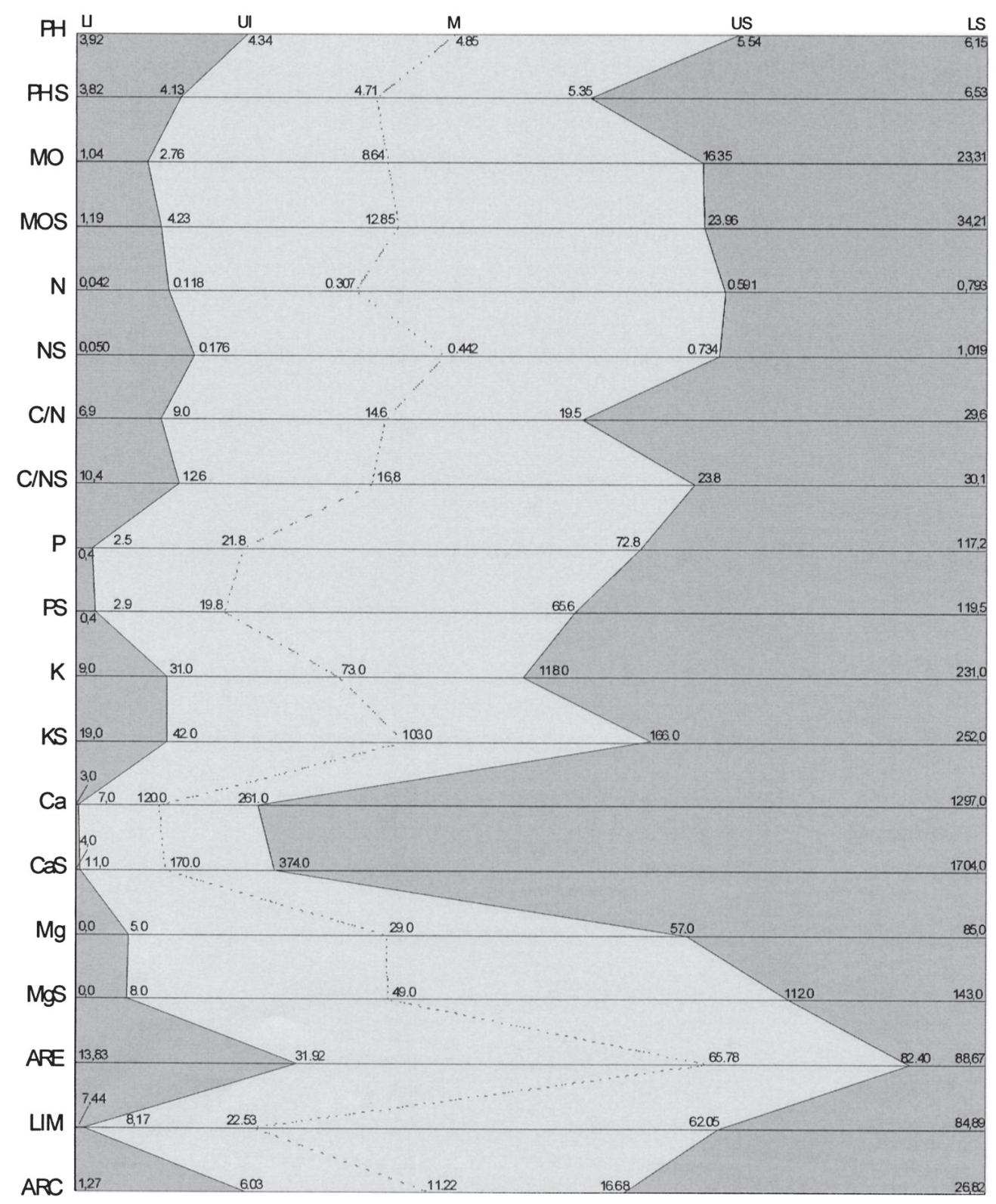

Figure 4. Habitat édaphique central et marginal des chênaies galiciennes de Quercus robur (voir liste des paramètres dans l'annexe).

nous avons utilisé tous les paramètres, 28 définissant le biotope et 14 dendrométrique/sylvicoles, car nous considérons que n'importe lequel d'entre eux peut posséder un caractère différenciateur dans la zone occupée par Quercus robur $[6,11,18$, $23,24,40]$. Dans le tableau IV on a rapporté les douze groupes résultants de l'application de la méthode Twinspan avec l'indication des parcelles et les paramètres discriminants.

Pour désigner parmi les paramètres sylvicoles utilisés celui qui explique le mieux la situation actuelle des forêts inventoriées et sa relation avec les paramètres du biotope, nous avons réalisé deux types d'analyses bivariables. Dans le tableau V sont présentés les coefficients de corrélation de Pearson des paramètres sylvicoles entre eux, avec leur niveau de signification et dans le tableau VI les coefficients correspondant à la confrontation de ces paramètres avec les descripteurs du biotope obtenus par la méthode Twinspan.

En dernier, pour expliquer la plus grande partie de variabilité possible de la population, nous avons réalisé quatre ACP [21, $39,61]$, incluant dans chacune d'entre elles comme variables de départ les quatre paramètres écologiques (ALT, PRO, TM, et TmA) que l'analyse de corrélations révèle comme les plus en relation avec les paramètres dendrométrique/sylvicoles, avec chacune de ces derniers (DMA, DMC, HMA et HMC). Le résultat fut semblable dans les quatre analyses, soit une 
Tableau III. Moyennes, déviations standards (DE), coefficients de variation (CV), valeur maximale et valeur minimale des paramètres dendrométriques/sylvicoles des forêts de Quercus robur $(n=39)$. Les variables sans unités sont adimensionnelles.

\begin{tabular}{|c|c|c|c|c|c|}
\hline Paramètre & Moyenne & $\mathrm{DE}$ & $\mathrm{CV}(\%)$ & Maximale & Minimale \\
\hline $\operatorname{DEN}\left(\mathrm{No} \mathrm{ha}^{-1}\right)$ & 935,19 & 473,66 & 50,65 & 2058,98 & 333,33 \\
\hline $\operatorname{ABA}\left(\mathrm{m}^{2} \mathrm{ha}^{-1}\right)$ & 57,0043 & 41,2624 & 72,38 & 171,8132 & 11,1362 \\
\hline DMA $(\mathrm{cm})$ & 27,38 & 10,13 & 36,99 & 46,38 & 12,10 \\
\hline $\mathrm{DMC}(\mathrm{cm})$ & 29,09 & 10,66 & 36,64 & 50,29 & 12,50 \\
\hline $\mathrm{DED}(\mathrm{cm})$ & 9,38 & 4,43 & 47,23 & 24,35 & 2,88 \\
\hline CVD (\%) & 34,74 & 10,93 & 31,46 & 55,34 & 17,49 \\
\hline $\mathrm{DOM}(\mathrm{cm})$ & 42,90 & 14,37 & 33,50 & 70,11 & 20,55 \\
\hline HMA (m) & 15,33 & 4,09 & 26,68 & 25,10 & 7,87 \\
\hline $\mathrm{HMC}(\mathrm{m})$ & 15,75 & 4,15 & 26,35 & 25,13 & 7,97 \\
\hline DEA (m) & 3,31 & 0,83 & 25,07 & 4,99 & 1,74 \\
\hline CVA $(\%)$ & 21,86 & 4,88 & 22,35 & 31,44 & 15,22 \\
\hline HDA (m) & 17,96 & 4,43 & 24,66 & 28,87 & 9,28 \\
\hline IHA $(\%)$ & 20,75 & 6,19 & 29,83 & 33,42 & 10,14 \\
\hline $\mathrm{ICZ}$ & 22,32 & 15,64 & 70,07 & 71,00 & 6,92 \\
\hline
\end{tabular}

Voir liste des paramètres dans l'annexe.

Tableau IV. Groupes finaux résultants de l'application du programme Twinspan.

\begin{tabular}{lcc}
\hline Groupe & Paramètres discriminants & Parcelles \\
\hline A & TMA $>$ M + DE & 28,29 y 30 \\
B & $\mathrm{DM}>\mathrm{M}+\mathrm{DE}$ & 6,8 y 39 \\
$\mathrm{C}$ & $\mathrm{DM}<\mathrm{M}+\mathrm{DE}$ & 4,11 y 23 \\
$\mathrm{D}$ & $\mathrm{DM}>\mathrm{M}+\mathrm{DE}$ & 5,31 y 35 \\
$\mathrm{E}$ & $\mathrm{PT}<\mathrm{M}+\mathrm{DE}$ & 19,20 y 21 \\
$\mathrm{~F}$ & $\mathrm{PT}>\mathrm{M}+\mathrm{DE}$ & 14 \\
$\mathrm{G}$ & $\mathrm{PRO}<\mathrm{M}-\mathrm{DE}$ & $13,16,25,33$ y 36 \\
& $\mathrm{PRO}>\mathrm{M}+\mathrm{DE}$ & 9 y 37 \\
$\mathrm{H}$ & $\mathrm{M}-\mathrm{DE}<\mathrm{PRO}<\mathrm{M}+\mathrm{DE}$ & $1,2,18,22,24,26,32,34$ y 38 \\
$\mathrm{I}$ & $\mathrm{TmA}>\mathrm{M}+\mathrm{DE}$ & 3,10 y 12 \\
$\mathrm{~J}$ & $\mathrm{PND}<\mathrm{M}+\mathrm{DE}$ & 17 \\
$\mathrm{~K}$ & $\mathrm{PND}>\mathrm{M}+\mathrm{DE}$ & 7,15 y 27 \\
& $\mathrm{ALT}<\mathrm{M}-\mathrm{DE}$ & \\
$\mathrm{L}$ & $\mathrm{M}-\mathrm{DE}<\mathrm{ALT}<\mathrm{M}+\mathrm{DE}$ & $\mathrm{ME}<\mathrm{DM}<\mathrm{M}+\mathrm{DE}$
\end{tabular}

description identique des quatre nouvelles variables créées. Dans les tableaux VII et VIII sont résumés, en exemples, les résultats de l'analyse menée à bien parmi les paramètres écologiques et le diamètre moyen quadratique.

\section{DISCUSSION}

En nous basant sur la description statistique univariable des paramètres du biotope (Tab. II) nous pouvons souligner que les plus importantes variabilités, avec des coefficients de variation supérieurs aux $70 \%$ apparaissent dans les paramètres PND,
TmA, P, PS, Ca, CaS, Mg, MgS et LIM. Dans le cas de la pente, cette donnée nous indique la situation actuelle de nombreuses chênaies dans des zones de coteaux accusés où elles ont perduré, car leur exploitation était très compliquée étant donné les caractéristiques physiographiques du milieu [28, 52]. Le coefficient élevé de variation du paramètre TmA interpelle particulièrement, c'est l'unique paramètre climatique avec un coefficient de variation supérieur à $40 \%$. Cependant, il est nécessaire de relever que les forêts inventoriées se trouvent à l'intérieur d'un ample domaine d'étude, la Galice, avec un rang thermique élevé, encore plus accentué, dû à l'ample variation altitudinale existante $[18,56]$. La haute variabilité de certains paramètres édaphiques ne surprend pas non plus, si ou prend en compte la diversité de la composition lithologique à l'intérieur du territoire galicien $[56,60]$. En contre position, les paramètres les plus homogènes, avec un coefficient de variation inférieur à $30 \%$ sont : PRO, PT, TM, TMA, PH, PHS et C/NS. Ce qui en ressort c'est l'homogénéité des paramètres climatiques, à l'exception TmA, ce qui nous indique l'existence, en général, d'un climat humide avec une certaine influence continentale dans des zones déterminées, accentuée par l'altitude et avec des précipitations élevées. Les sols sont assez profonds puisqu'il s'agit d'une espèce avec un système racinaire fortement développé, avec une racine principale qui peut arriver à pénétrer jusqu'à $1,5 \mathrm{~m}[1,12]$. Il s'agit de sols acides avec des valeurs de $\mathrm{pH}$ qui présentent peu de variation.

Comme caractéristiques les plus notables de l'habitat physiographique central (Fig. 2) nous soulignons les suivantes dont certaines ont déjà été mises en évidence dans l'analyse univariable :

- La majeure partie des forêts se trouve dans des zones de pente élevée et dans des fonds de talweg, résultat de la pression anthropique à laquelle elles ont été soumises [28, 52], occupant des lieux de basse et moyenne altitude, avec des orientations variables et une préférence des orientations à l'ombre [12,60] (Tab. I). 
Tableau V. Coefficients de corrélation linéaire de Pearson entre les paramètres dendrométriques/sylvicoles.

\begin{tabular}{|c|c|c|c|c|c|c|c|c|c|c|c|c|c|c|}
\hline & DEN & $\mathrm{ABA}$ & DMA & DMC & DED & CVD & DOM & HMA & HMC & DEA & CVA & HDA & IHA & ICZ \\
\hline DEN & 1,000 & n.s. & $-0,513 * *$ & $-0,551 * *$ & $-0,624 * *$ & $-0,430 * *$ & $-0,476 * *$ & $-0,438^{*}$ & $-0,469 * *$ & n.s. & n.s. & $-0,374 *$ & $-0,502 * *$ & n.s. \\
\hline ABA & & 1,000 & $0,833^{* *}$ & $0,793 * *$ & $0,354^{*}$ & n.s. & $0,791 * *$ & $0,641 * *$ & $0,627 * *$ & n.s. & n.s. & $0,605^{* *}$ & $-0,543 * *$ & $0,616^{* *}$ \\
\hline DMA & & & 1,000 & $0,995 * *$ & $0,694 * *$ & n.s. & $0,959 * *$ & $0,756^{* *}$ & $0,774 * *$ & $0,336^{*}$ & n.s. & $0,682 * *$ & n.s. & n.s. \\
\hline DMC & & & & 1,000 & $0,764 * *$ & n.s. & $0,969 * *$ & $0,732 * *$ & $0,757 * *$ & $0,360 *$ & n.s. & $0,662 * *$ & n.s. & n.s. \\
\hline DED & & & & & 1,000 & $0,660 * *$ & $0,777 * *$ & $0,385^{*}$ & $0,447 * *$ & $0,413^{*}$ & n.s. & $0,361 *$ & n.s. & n.s. \\
\hline CVD & & & & & & 1,000 & n.s. & n.s. & n.s. & n.s. & n.s. & n.s. & $0,581 * *$ & $-0,403^{*}$ \\
\hline DOM & & & & & & & 1,000 & $0,665^{* *}$ & $0,690^{* *}$ & $0,345^{*}$ & n.s. & $0,610 * *$ & n.s. & n.s. \\
\hline HMA & & & & & & & & 1,000 & $0,993 * *$ & $0,625^{* *}$ & n.s. & $0,954 * *$ & $-0,439 *$ & $0,674 * *$ \\
\hline HMC & & & & & & & & & 1,000 & $0,649 *$ & n.s. & $0,961 * *$ & $-0,411^{*}$ & $0,642 * *$ \\
\hline DEA & & & & & & & & & & 1,000 & n.s. & $0,674 * *$ & n.s. & $0,414 *$ \\
\hline CVA & & & & & & & & & & & 1,000 & n.s. & n.s. & n.s. \\
\hline HDA & & & & & & & & & & & & 1,000 & $-0,532 * *$ & $0,687 * *$ \\
\hline IHA & & & & & & & & & & & & & 1,000 & $-0,817 * *$ \\
\hline ICZ & & & & & & & & & & & & & & 1,000 \\
\hline
\end{tabular}

Niveaux de signification (s): $* \mathrm{~s}>95 \% ; * * \mathrm{~s}>99 \%$; n.s., non significatif.

Tableau VI. Coefficients de corrélation linéaire de Pearson entre les paramètres dendrométriques/sylvicoles et les paramètres descripteurs du biotope obtenus avec le programme Twinspan.

\begin{tabular}{lcccccccc}
\hline & ALT & PND & PRO & DM & PT & TM & TMA & TmA \\
\hline DEN & $0,355^{*}$ & n.s. & n.s. & n.s. & n.s. & n.s. & n.s. & $-0,359^{*}$ \\
ABA & n.s. & n.s. & $-0,352^{*}$ & n.s. & n.s. & n.s. & n.s. & $0,360^{*}$ \\
DMA & $-0,438^{* *}$ & n.s. & $-0,456^{* *}$ & n.s. & n.s. & $0,484^{* *}$ & n.s. & $0,540^{* *}$ \\
DMC & $-0,437^{* *}$ & n.s. & $-0,446^{* *}$ & n.s. & n.s. & $0,484^{* *}$ & n.s. & $0,524^{* *}$ \\
DED & n.s. & $0,346^{*}$ & n.s. & n.s. & n.s & $0,352^{*}$ & n.s. & n.s. \\
CVD & n.s. & $0,380^{*}$ & n.s. & n.s & n.s & n.s. & n.s. & n.s. \\
DOM & $-0,375^{*}$ & n.s. & $-0,460^{* *}$ & n.s. & n.s. & $0,407^{*}$ & n.s. & $0,430^{* *}$ \\
HMA & $-0,442^{*}$ & n.s. & $-0,368^{*}$ & n.s. & n.s. & $0,475^{* *}$ & n.s. & $0,468^{* *}$ \\
HMC & $-0,430^{*}$ & n.s. & $-0,356^{*}$ & n.s. & n.s. & $0,507^{* *}$ & n.s. & $0,479^{* *}$ \\
DEA & $-0,485^{* *}$ & n.s. & n.s. & n.s. & n.s & $0,517^{* *}$ & $0,471^{* *}$ & n.s. \\
CVA & n.s. & n.s. & n.s. & n.s & n.s. & n.s. & n.s. & n.s. \\
HDA & n.s. & n.s. & n.s. & n.s. & n.s. & $0,459^{* *}$ & n.s. & $0,381^{*}$ \\
IHA & n.s. & $0,393^{*}$ & n.s. & n.s. & n.s. & n.s. & n.s. & n.s. \\
ICZ & n.s. & n.s. & n.s. & n.s. & n.s. & n.s. & n.s. & n.s. \\
\hline
\end{tabular}

Niveaux de signification (s) : $* \mathrm{~s}>95 \% ; * * \mathrm{~s}>99 \%$; n.s., non significatif.

Tableau VII. Résumé du A.C.P. entre le diamètre moyen quadratique et les paramètres du biotope sélectionnés dans l'analyse de corrélations.

\begin{tabular}{|c|c|c|c|c|c|c|}
\hline \multirow{2}{*}{ Composant } & \multicolumn{3}{|c|}{ Autovecteurs initiaux } & \multicolumn{3}{|c|}{$\begin{array}{l}\text { Addition des saturations } \\
\text { aux quadrats de l'extraction }\end{array}$} \\
\hline & Total & $\%$ de la variance & $\%$ accumulé & Total & $\%$ de la variance & $\%$ accumulé \\
\hline 1 & 2,917 & 58,340 & 58,340 & 2,917 & 58,340 & 58,340 \\
\hline 2 & 0,879 & 17,575 & 75,915 & 0,879 & 17,575 & 75,915 \\
\hline 3 & 0,522 & 10,442 & 86,357 & 0,522 & 10,442 & 86,357 \\
\hline 4 & 0,494 & 9,879 & 96,235 & & & \\
\hline 5 & 0,188 & 3,765 & 100,000 & & & \\
\hline
\end{tabular}


Tableau VIII. Matrice de composants de l'A.C.P. du diamètre moyen quadratique avec les variables du biotope dans les forêts de Quercus robur.

\begin{tabular}{lrrr}
\hline Variables initiales & Comp. 1 & Comp. 2 & Comp. 3 \\
\hline Altitude (ALT) & $-0,773$ & $-0,488$ & 0,239 \\
Profondeur (PRO) & $-0,645$ & 0,605 & 0,433 \\
Température moyenne annuelle (TM) & 0,839 & 0,422 & 0,025 \\
Température moyenne annuelle minimas absolus (TmA) & 0,794 & $-0,233$ & 0,125 \\
Diamètre moyen quadratique (DMC) & 0,755 & $-0,207$ & 0,511 \\
\hline
\end{tabular}

- La profondeur du sol est élevée, ce qui provient de la présence de nombreuses forêts en zones de talweg, comme nous l'avons remarqué antérieurement, dû à l'existence dans ces zones de sols libres, frais et profonds particulièrement aptes pour Quercus robur [1, 12, 62].

- L'habitat central du paramètre distance à la mer est compris entre 12 et $79 \mathrm{~km}$ en ligne droite, ce qui correspond à la distribution potentielle de la végétation en Galice [37, 38, 53].

Quant à l'habitat physiographique marginal (Fig. 2), il faut remarquer l'existence de chênaies dans des territoires relativement éloignés de leur zone potentielle [37, 38, 53, 60], donc, dans ces cas il apparaît souvent hybridé avec d'autres espèces du genre Quercus, comme $Q$. petraea dans les parcelles de Ancares et Serra do Invernadeiro et $Q$. pyrenaica dans la parcelle de Valdín. Ces parcelles subissent une grande influence continentale et se trouvent à une altitude élevée, caractéristiques non optimum pour la présence de $Q$. robur $[1,18,62]$.

De l'analyse de l'habitat climatique central (Fig. 3) nous pouvons souligner ce qui suit :

- La majeure partie des chênaies se trouvent dans des zones avec des précipitations annuelles proches ou supérieures à $1000 \mathrm{~mm}$ avec une précipitation estivale entre 100 et $200 \mathrm{~mm}$, ce qui indique des besoins élevés en humidité de l'environnement $[1,12,62]$. Les précipitations suivent dans la majeure partie des parcelles la séquence [18]: automne $>$ hiver $>$ printemps $>$ été.

- Quant au régime thermique, il existe une faible amplitude dans les valeurs des paramètres thermiques, sauf pour la TmA. Comme nous l'avons commenté antérieurement, les forêts inventoriées se situent dans un domaine d'étude très vaste, avec une variation thermique élevée, encore plus accusée du fait de l'ample variation altitudinale existante $[5,51,56]$.

Vis-à-vis de l'habitat climatique marginal (Fig. 3), il faut remarquer uniquement la présence de chênaies dans des zones avec des précipitations estivales faibles (facteur qui ne favorise pas le développement de l'espèce $[1,12,62]$ ) mais compensées dans ces cas par des brumes et brouillards. Il s'agit de forêts qui se trouvent dans des zones de Galice avec une certaine influence méditerranéenne, principalement dans la province d'Ourense (Fig. 1).

Si nous analysons les valeurs qui limitent l'habitat édaphique central (Fig. 4) nous pouvons souligner les caractéristiques suivantes :

- La composition granulométrique de la terre fine présente une amplitude remarquable dans le pourcentage de sable et de limon et beaucoup moins importante pour l'argile. Dans l'ensemble, les sols peuvent être considérés de texture franche et suffisamment sableuse. Ce facteur, avec le fait que beaucoup de parcelles se trouvent dans des zones de forte pente, peut donner lieu à une légère érosion, avec une lente perte d'éléments fins entraînés vers le bas du coteau. Ce phénomène existe malgré l'existence d'un puissant système racinaire du chêne, et est accentué dans les zones de fortes rocailles [11].

- Le pourcentage de matière organique oscille entre une valeur minimale de $1,04 \%$ pour le total du profil et de $1,19 \%$ pour les $20 \mathrm{~cm}$ supérieurs, jusqu'à une valeur maximale de $23,31 \%$ et de $34,21 \%$ respectivement. La composition granulométrique existante, unie aux valeurs modérées de matière organique, donne origine à des sols avec une faible capacité de cimentation $[11,23]$.

- La lithologie est de nature siliceuse avec de sols d'une acidité élevée, dominant le type cambisol et restant enclavés dans les classes très fortement et fortement acides dans l'échelle d'acidité de [64]. La relation $\mathrm{C} / \mathrm{N}$ est relativement constante, aussi bien dans l'ensemble du profil, où elle oscille entre 9,0 et 19,5, qu'en surface, où elle est légèrement plus élevée, oscillant entre 12,6 et 23,8. Malgré ceci, à cause des basses valeurs de $\mathrm{pH}$, nous n'arrivons pas aux conditions optimales d'humification, c'est pourquoi l'humus est de type mull-moder [11].

- La fertilité du sol, exprimée par la richesse en nutriments, peut être considérée comme relativement basse ou moyenne, même si la concentration en phosphore est remarquablement supérieure à celle rapportée par d'autres auteurs comme Castroviejo [11], dû possiblement à ce que certaines des chênaies se trouvent dans des zones occupées par d'anciennes cultures [27, 28]. La teneur en potassium s'inclut dans les besoins optimum [23].

La description statistique univariable des paramètres dendrométriques et sylvicoles (Tab. III) nous indique la haute variabilité des paramètres $\mathrm{DEN}, \mathrm{ABA}$ et ICZ avec un coefficient de variation supérieur à $50 \%$. Dans le cas du reste des paramètres en relation avec le diamètre normal (DMA, DMC, DED, CVD et DOM) et particulièrement avec la hauteur totale (HMA, HMC, DEA, CVA, HAD et IHA), la variabilité est moindre avec des valeurs du coefficient de variation plus réduites [6, 18].

La majeure partie des forêts inventoriées provient de taillis et, il serait nécessaire de les convertir en futaie régulière en vue d'une production de bois de qualité [19]. Bien sûr il est également possible d'atteindre cet objectif dans les forêts semi-régulières, mais celles-ci, présentent un aménagement plus complexe [7, 40]. Le développement d'une sylviculture productive pour 
Quercus robur en Galice ne peut être pensé que pour des endroits qui présentent une fertilité de station mi-haute [6]. D'un autre côté, le niveau de gestion que ceci supposerait n'a jamais été appliqué dans les chênaies galiciennes, sans compter sur l'expérience préalable dans l'application de certaines prescriptions sylvicoles nécessaires, comme la désignation d'arbres d'élite ou l'application d'éclaircies sélectives. La croyance dans la difficulté des traitements sylvicoles à appliquer et la croissance lente de l'espèce a conduit dans beaucoup de cas à l'abandon des forêts ou à leur remplacement par des espèces de croissance rapide, tels que, conifères et eucalyptus [6, 18, 19].

Les paramètres discriminants obtenus dans la caractérisation de groupes correspondent dans tous les cas à des paramètres physiographiques (ALT, PND, PRO et DM), ou bien climatiques (PT, TM, TMA et TmA), sans influencer le reste des paramètres que nous avons considérés dans l'analyse, ce qui pourrait indiquer que la distribution des chênaies en Galice est beaucoup plus en relation avec la physiographie et le climat qu'avec les conditions édaphiques $[6,11,18]$.

De l'ensemble des 14 paramètres sylvicoles considérés, ceux qui sont les plus significativement en relation avec tous les autres sont (Tab. V) : DMA, DMC, HMA, HMC et HDA. Donc, ils seraient les plus indiqués pour les confronter avec les paramètres descripteurs du biotope (ALT, PND, PRO, DM, PT, TM, TMA, et TmA). De ceux-ci, il faut éliminer le paramètre de hauteur dominante (HDA), car il se trouve très influencé par différents traitements sylvicoles malencontreux appliqués à beaucoup de ces forêts (élagages et têtards pour l'alimentation du bétail) [27, 28, 50,52]. Une fois sélectionnés les quatre paramètres dendrométrique/sylvicoles, nous observons dans le tableau VI que tous ceux-ci sont beaucoup en relation avec les suivants paramètres descripteur du biotope : ALT, PRO, TM et TmA, et même, dans certain cas, comme il arrive avec DMA et DMC $(\mathrm{s}=99 \%)$. Donc, nous considérons que tous ceux-ci sont adéquats pour définir correctement les caractéristiques sylvicoles des chênaies galiciennes de Quercus robur $[6,18$, 62]. Du reste des paramètres dendrométrique/sylvicoles se détachent les valeurs du coefficient de corrélation de Pearson obtenues entre la DEA et les paramètres écologiques discriminants, ALT, TM et TmA, avec des niveaux de signification de $99 \%$; cependant, ce paramètre ne semble pas adéquat pour le choisir comme définissant la situation sylvicole actuelle des chênaies $[9,25,55]$.

Comme on peut le vérifier dans les tableaux VII et VIII, l'utilisation de la méthodologie ACP est très adéquate pour la population de données maniée, car dans tous les cas, la moyenne d'adéquation de Kaiser-Meyer-Olkin (KMO) s'approche de 0,7 , avec une signification du résultat de l'analyse très élevée $[21,61]$. De cette façon, il est possible d'expliquer plus de $86 \%$ de la variabilité des chênaies avec seulement trois nouvelles variables ou vecteurs (Tab. VII). La première de ces variables expliquerait dans tous les cas, à peu près $58 \%$ de la variabilité, et à travers l'interprétation de la matrice de composants de l'analyse (Tab. VIII), on identifie clairement la température, spécialement la TM. Dans un second temps, en expliquant entre 17,5 et $18 \%$ de la variabilité se situe une seconde variable, dans la composition de laquelle participe majoritairement la PRO. Enfin, dans les quatre analyses présentées, un troisième vecteur apparaît qui donne des explications correspondant un peu plus de $10 \%$ de la variabilité totale, avec ceci on atteint $86 \%$. Ce troisième vecteur s'identifie (Tab. VIII) avec chacun des paramètres dendrométrique/sylvicoles utilisés dans chaque cas. En résumé, on peut conclure que la variabilité présente dans les forêts de Quercus robur inventoriées peut être expliquée dans plus de $86 \%$ du total par trois variables identifiées comme la température moyenne annuelle de la station où se trouve chaque forêt, la profondeur du sol et le paramètre dendrométrique/sylvicole incorporé dans chaque cas à l'analyse (Tab. VII). Ces résultats sont similaires à ceux obtenus chez d'autres auteurs $[6,11,18]$, lesquels signalent que la distribution des chênaies en Galice est beaucoup plus en relation avec les caractéristiques physiographiques et climatiques qu'avec les conditions édaphiques du territoire.

Remerciements : Ce travail a été financé par la « Dirección Xeral de Investigación e Desenvolvemento da Consellería de Innovación, Industria e Comercio da Xunta de Galicia » dans le cadre des projets de recherche PGIDT99MA29101 et PGIDIT02RFO29101PR développés par le Département d'Enxeñería Agroforestal de l'Université de Santiago de Compostela.

\section{ANNEXE : Liste des paramètres élaborés}

Altitude moyenne (ALT), pente moyenne (PND), profondeur du sol jusqu'à la roche mère (PRO), distance de la mer en ligne droite (DM), précipitation totale annuelle (PT), précipitation estivale (PE), température moyenne annuelle (TM), température moyenne annuelle des maximas absolus (TMA), température moyenne annuelle des minimas absolus (TmA), sable total (ARE), limon total (LIM), argile totale (ARC), $\mathrm{pH}$ total $\mathrm{H}_{2} \mathrm{O}(\mathrm{PH})$, pH superficiel $\mathrm{H}_{2} \mathrm{O}$ (PHS), matière organique totale (MO), matière organique superficielle (MOS), azote total $(\mathrm{N})$, azote superficiel (NS), rapport carbone azote total $(\mathrm{C} / \mathrm{N})$, rapport carbone azote superficiel (C/NS), phosphore assimilable total (P), phosphore assimilable superficiel (PS), potassium échangeable total $(\mathrm{K})$, potassium échangeable superficiel $(\mathrm{KS})$, calcium échangeable total $(\mathrm{Ca})$, calcium échangeable superficiel $(\mathrm{CaS})$, magnésium échangeable total $(\mathrm{Mg})$, magnésium échangeable superficiel $(\mathrm{MgS})$, nombre de pieds par hectare $(\mathrm{DEN})$, surface terrière par hectare $(\mathrm{ABA})$, diamètre moyen arithmétique (DMA), diamètre moyen quadratique (DMC), déviation standard de la distribution diamétrale (DED), coefficient de variation de la distribution diamétrale (CVD), diamètre dominant (DOM), hauteur moyenne arithmétique (HMA), hauteur moyenne quadratique (HMC), déviation standard de la distribution de hauteurs (DEA), coefficient de variation de la distribution de hauteurs (CVA), hauteur dominante de Assmann (HDA), indice de Hart (IHA), indice de Czarnowski (ICZ).

\section{RÉFÉRENCES}

[1] Amaral J., Quercus, in: Castroviejo S. et al. (Eds.), Flora Ibérica II, C.S.I.C., Madrid, 1990, pp. 15-36.

[2] Assmann E., The principles of forest yield study, Pergamon Press, Oxford, New York, 1970. 
[3] Aramburu M.P. Escribano R., Martínez E., Sáenz D., Análisis de la distribución de Quercus pyrenaica Willd en el Sistema Central, Fundación Conde del Valle de Salazar, E.T.S.I.M., Madrid, 1984.

[4] Ashton M.S., Larson B.C., Germination and seedling growth of Quercus (section Erythrobalanus) across openings in a mixed-deciduous forest of southern New England, USA, For. Ecol. Manage. 80 (1996) 81-94.

[5] Aussenac G., Interactions between forest stands and microclimate: Ecophysiological aspects and consequences for silviculture, Ann. Sci. For. 57 (2000) 287-301.

[6] Barrio M., Crecimiento y producción de masas naturales de Quercus robur L en Galicia, Tesis doctoral, Univ. Santiago de Compostela (inédito), 2003.

[7] Bary-Lenger A., Nebout J.P., Le chêne pédonculé et sessile en France et en Belgique, Gerfaut Club Editions du Perron, AlleurLiège, 2003.

[8] Bellot F., La vegetación de Galicia, Anales del Instituto Botánico A. J. Cavanilles XXIV, C.S.I.C., Madrid, 1966.

[9] Blanco A., Rubio A., Sánchez O., Elena R., Gómez V., Graña D., Autoecología de los castañares de Galicia (España), Investig. Agrar. Sist. Recur. For. 9 (2000) 337-361.

[10] Carballeira, A., Devesa, C., Retuerto, R., Santillan, E., Ucieda, F. Bioclimatología de Galicia, Xunta de Galicia-Fundación Barrie de la Maza, 1983.

[11] Castroviejo M., Fitoecología de los montes de Buio y Sierra del Xistral (Lugo), Xunta de Galicia, Velograf, Santiago de Compostela, 1988.

[12] Ceballos L., Ruíz de la Torre J., Árboles y arbustos, E.T.S.I., Madrid, 1979.

[13] Daget P., Godron M., Analyse fréquentielle de l'écologie des espèces dans les communautés, Masson, Paris, 1982.

[14] Dantas R.R., Os carvalhais da Serra da Peneda. Estudio fitosociológico, Agron. Lusit. 20 (1958) 83-152.

[15] Díaz T.E., Fernández J.A., Itinera Geobotánica 8 (1994) 243-528.

[16] Díaz-Fierros F., Calvo de Anta R., Paz A., As especies forestais e os solos de Galicia, Cuad. Área Cienc. Agrarias, Sem. Est. Gall. 3 (1982) 15-30.

[17] Díaz-Fernández P.M., Jiménez P., Martín S., De Tuero M., Gil L., Regiones de procedencia de Quercus robur L., Q. petraea (Matt) Liebl y $Q$. humilis Miller, Instituto de Conservación de la Naturaleza, Madrid, 1995.

[18] Díaz-Maroto I.J., Estudio ecológico y dasométrico de las masas de carballo (Quercus robur L.) en Galicia, Tesis doctoral, Univ. Politécnica de Madrid (inédito), 1997.

[19] Díaz-Maroto I.J., Silva-Pando F.J., Ordenación de masas de Quercus robur L. en el noroeste peninsular, Cuadernos S.E.C.F. 1 (1995) 211-219.

[20] DGCONA, Tercer Inventario Forestal Nacional 1997-2006: Galicia, Ministerio Medio Ambiente, Dirección de Conservación de la Naturaleza, 2001.

[21] Gabriel K.R., Biplot, in: Kotz S., Johnson N.L. (Eds.), Encyclopedia of Statistical Sciences, Vol. 1, Wiley, New York, 1982, pp. 263-271.

[22] Gaines S.D., Denny M.W., The largest, smallest, highest, lowest, longest and shortest: extremes in ecology, Ecology 74 (1993) $1677-1692$.

[23] Gandullo J.M., Sánchez O., González S., Estudio ecológico de las tierras altas de Asturias y Cantabria, Monografías I.N.I.A. 49, Madrid, 1983.

[24] Gandullo J.M., Bañares A., Blanco A., Castroviejo M., Fernández López A., Muñoz L., Sánchez O., Serrada R., Estudio ecológico de la laurisilva canaria, Instituto de Conservación de la Naturaleza, Madrid, 1991.
[25] Gómez V., Blanco A., Sánchez O., Rubio A., Elena R., Graña D. Autoecología de los castañares andaluces, Investig. Agrar. Sist. Recur. For. 11 (2002) 205-226.

[26] Greig-Smith P., Quantitative plant ecology, Blackwell Scientific Publications, Oxford, 1983.

[27] Guitián Rivera L., Sistemas de utilización del espacio y evolución del paisaje vegetal en las Sierras Orientales de Lugo, in: Pérez Alberti et al. (Eds.), La evolución del paisaje en las montañas del entorno de los Caminos Jacobeos, Cambios ambientales y actividad humana, Xunta de Galicia, 1993, pp. 211-225.

[28] Guitián Rivera L., Origen y evolución de la cubierta forestal de Galicia, Tesis doctoral, Univ. Santiago de Compostela (inédito), 1995.

[29] Hart H.M.F., Stamtal en dunning ; een orienteerend onderzoek naar de beste plantwijdte en duningswijze loor den djati, Veenman \& Zonen, 1928

[30] Hill M.O., Reciprocal averaging: An eigenvector method of ordination, J. Ecol. 61 (1973) 237-249.

[31] Hill M.O., Decorana: A Fortran program for detrended correspondence analysis and reciprocal averaging, Cornell Univ., New York, 1979.

[32] Hill M.O., Twinspan: A Fortran program for arranging multivariate data in an ordered two-way table by classification of the individuals and attributes, Cornell Univ., New York, 1979.

[33] Hill M.O., Bunce R.G.H., Shaw M.W., Indicator species analysis: a divisive polythetic method of classification, and its application to a survey of native pinewoods in Scotland, J. Ecol. 63 (1975) 597613.

[34] Hix D.M., Multifactor classification and analysis of upland hardwood forest ecosystems of the Kichapoo River watershed, southwestern Wisconsin, Can. J. For. Res. 18 (1988) 1405-1415.

[35] Hochbichler E., Methods of oaks silviculture in Austria, Ann. Sci. For. 50 (1993) 583-591.

[36] Humphrey J.W., Swaine M.D., Factors affecting the natural regeneration of Quercus in Scottish oakwoods. Part I. Competition from Pteridium aquilinum, J. Appl. Ecol. 34 (1997) 577-524.

[37] Izco J., Galicia, in: Peinado M., Rivas-Martínez S. (Eds.), La vegetación de España, Univ. de Alcalá de Henares, Madrid, 1987, pp. 354-415.

[38] Izco J., Amigo J., Guitian J., Los robledales galaico-septentrionales, Acta Bot. Malacit. XV (1990) 267-276.

[39] Jobson J.D., Applied multivariate data analysis, Springer-Verlag, 1991.

[40] Johnson P.S., Shifley S.R., Rogers R., The ecology and silviculture of oaks, CABI Publishing, Wallingford, 2002.

[41] Kelly D.L., The regeneration of Quercus petraea (sessile oak) in southwest Ireland: a 25-year experimental study, For. Ecol. Manage. 166 (2002) 207-226.

[42] Kenk G.K., New perspectives in German oak silvicultura, Ann. Sci. For. 50 (1993) 563-570

[43] Kent M., Coker P., Vegetation description and analysis. A practical approach, John Wiley \& Sons, New York, 1996.

[44] Lebourgeois F., Cousseau G., Ducos Y., Climate-tree-growth relationships of Quercus petraea Mill. stand in the forest of Bercé ("Futaie des Clos", Sarthe, France), Ann. For. Sci. 31 (2004) 361372.

[45] Losa J.M., Estudio de las comunidades arbóreas naturales de la cuenca media del río Eume (A Coruña), Trab. Compostel. Biol. 3 (1973) $1-63$

[46] Losa J.M., Vegetación y flora de la cuenca del río Eume. Estudio botánico y fitosociológico del bosque natural de Quercus robur L., Bol. Soc. Broter. 47 (1974) 243-267. 
[47] Martínez J.E., Ayuga E., González C., Estudio comparativo de distintas funciones núcleo para la obtención del mejor ajuste según el tipo de datos, Qüesttió 16 (1992) 3-26.

[48] Pardé J., Bouchon J., Dendrométrie, E.N.G.R.E.F., 2e ed., Nancy, 1988.

[49] Pisces Conservation L.T.D., Community Analysis Package versión 1.42. A program to search for structure in ecological community data, England, 1999.

[50] Pulido F.J., Biología reproductiva y conservación: el caso de la regeneración de bosques templados y subtropicales de robles (Quercus spp.), Rev. Chil. Hist. Nat. 75 (2002) 5-15.

[51] Retuerto R., Carballeira A., Phytoecological importance, mutual redundancy and phytological threshold values of certain climatic factors, Vegetatio 90 (1990) 47-62.

[52] Rigueiro A., Aspectos botánicos e ecolóxicos das frondosas caducifolias autóctonas de interese para Galicia, in: Rigueiro et al. (Eds.), Repoboación con frondosas caducifolias en Galicia, Univ. Santiago. de Compostela, 1995, pp. 5-29.

[53] Rivas-Martínez S., Memoria y mapas de series de vegetación de España. 1:400.000, Instituto de Conservación de la Naturaleza, Madrid, 1987.

[54] Röhrig E., Ulrich B., Ecosystems of the world, 7. Temperate Decidous Forests, Elsevier Science Publisers Company Inc., New York, 1991.
[55] Rubio A., Escudero A., Gandullo J.M., Sweet chestnut silviculture in a ecological extreme of its range in the west of Spain (Extremadura), Ann. Sci. For. 54 (1997) 667-680.

[56] Ruíz de la Torre J., Mapa Forestal de España. Varias hojas, Dirección General de Conservación de la Naturaleza, Madrid, 1991.

[57] Russell J.S., Moore A.W., Comparison of different depth weightings in the numerical analysis of anisotropic soil profile data, Proc. 9th. Int. Cong. Soil Sci. 4 (1968) 205-213.

[58] Ryan T.P., Modern regression methods, John Wiley \& Sons, 1997.

[59] Schütz J.P., Sylviculture 1. Principes d'Éducation des Forêts, Presses polytechniques et universitaires romandes, Lausanne, 1990.

[60] Silva-Pando F.J., Rigueiro A., Guía das árbores e bosques de Galicia, Galaxia, Vigo, 1992.

[61] Ter Braak C.J.F., Canonical community ordination. Part I: Basic theory and linear methods, Ecoscience 1 (1994) 127-140.

[62] Timbal J., Aussenac G., An overview of ecology and silviculture of indigenous oaks in France, Ann. Sci. For. 53 (1996) 649-661.

[63] Walpole R.E., Myers R.H., Myers S.L., Probabilidad y estadística para ingenieros, 6th ed., Prentice Hall, Londres, 1999.

[64] Wilde S.A., Forest soils and forest growth, Chronica Botanica Comp., 1946.

[65] Xunta de Galicia, Avance do Segundo Inventario Forestal Nacional 1986. Consellería de Agricultura, Gandería e Montes, Dirección Xeral do Forestal e do Medio Ambiente Natural, Santiago de Compostela, 1988. 